\title{
OPTICAL FOLLOW-UP OBSERVATIONS OF ASCA LYNX DEEP SURVEY
}

K. OHTA, M. AKIYAMA, K. NAKANISHI

Dept. Astronomy, Kyoto Univ., Kyoto 606-01, Japan

T. YAMADA

Astronomical Institute, Tohoku Univ., Sendai 980-77, Japan

K. HAYASHIDA

Dept. Earth and Space Science, Osaka Univ., Osaka 560, Japan

T. KII

Institute of Space and Astronautical Science, Kanagawa 229, Japan

AND

Y. OGASAKA

NASA/GSFC, Greenbelt, MD 20771, USA

\section{Introduction}

Since the bulk of the energy density of the Cosmic X-ray Background (CXB) resides in the harder energy band than that of the ROSAT band (0.5-2 $\mathrm{keV}$ ) and since the X-ray sources identified in the ROSAT band have X-ray spectra softer than that of the $\mathrm{CXB}$, investigation of nature of the X-ray sources at the harder energy band is indispensable to solve the origin of the CXB. However, only $2-3 \%$ of the CXB in the hard band (2-10 keV) had been resolved into discrete sources (Piccinotti et al. 1982, ApJ 253, 485). We present our preliminary results of optical follow-up observations of the ASCA Lynx deep survey.

\section{X-ray observations}

The X-ray observations were made with the ASCA with an exposure time of about $80 \mathrm{ksec}$. The flux limit in the $2-10 \mathrm{keV}$ band is more than 100 times deeper than that of the previous survey. The observed field is Lynx 
$3 \mathrm{~A}$ and the field of view of SIS is about $20^{\prime}$ by $20^{\prime}$. Details of the X-ray observations are presented in Ogasaka (1997, in this proceedings). We define an X-ray source sample containing 8 sources by selecting X-ray sources detected above $5.5 \sigma$ confidence. The fluxes of the sources range from $3 \times$ $10^{-14}$ to $12 \times 10^{-14} \mathrm{erg} \mathrm{s}^{-1} \mathrm{~cm}^{-2}$ in $0.7-7 \mathrm{keV}$ band and from $5 \times 10^{-14}$ to $10 \times 10^{-14} \mathrm{erg} \mathrm{s}^{-1} \mathrm{~cm}^{-2}$ in $2-7 \mathrm{keV}$ band.

\section{Optical follow-up observations}

Optical imaging observations were made in R-band with the Kiso Schmidt $(1.05 \mathrm{~m})$ in Japan and in I-band with the University of Hawaii $2.2 \mathrm{~m}$ and $0.6 \mathrm{~m}$ telescopes. We picked up candidates of optical counterpart in an error circle (radius $\sim 30^{\prime \prime}$ ) of an ASCA source. In this process, we also used the ROSAT SRC catalogue, in which X-ray sources are cataloged with a typical error radius of about $10^{\prime \prime}$. Note that all the sources identified below are also ROSAT sources. Optical spectroscopic observations were carried out for these candidates using the KPNO $2.1 \mathrm{~m}$ with the Gold Camera and the KPNO 4m with the Cryo Cam.

\section{Results}

Two sources are identified with type-1 (broad emission line) AGNs with redshifts of 0.46 and 0.57 . One type 1.5 AGN is also identified at $z=0.56$. These objects have X-ray luminosities of $(1-7) \times 10^{44} \mathrm{erg} \mathrm{s}^{-1}(2-10 \mathrm{keV})$. We have two more type-1 AGN counterparts, but only one broad emission line is seen in the spectra; possible redshifts of these objects are 4.2, 3.2, and 1.3, if the emission is Ly $\alpha, \mathrm{CIV}$, and MgII, respectively. Other $3 \mathrm{X}$-ray sources have not yet optically identified. However since one of them shows an excess number density of faint galaxies, it may be a cluster of galaxies.

Our $5.5 \sigma$ sample has total flux of $3.95 \times 10^{-13} \mathrm{erg} \mathrm{s}^{-1} \mathrm{~cm}^{-2}$ in the 2 - $7 \mathrm{keV}$ band which corresponds to about $27 \%$ of the CXB in this band. Type-1 AGNs/QSOs including type-1.5 and the objects without the certain redshift contribute $19 \%$ of the CXB in this band. The possible cluster has about $4 \%$ contribution.

It should be worth noting that in course of the follow-up program, we obtained optical spectra for less significant X-ray sources. One of which is AX J08494+4454. This object was identified with a type-2 QSO at $z=0.9$ (Ohta et al. 1996, ApJ 458, L57) and contributes about $7 \%$ of the CXB in the $2-7 \mathrm{keV}$ band. 